\title{
SOME REMARKS ON NON-COMMUTATIVE EXTENSIONS OF LOCAL RINGS
}

\author{
EDWARD H. BATHO
}

In $[1]^{*}$ we introduced the concept of a non-commutative local ring and studied the structure of such rings. Unfortunately, we were not able to show that the completion of a local ring was a semi-local ring. In this paper we propose to study a class of rings for which the above result is valid. This class of rings is the integral extensions-[4,5]-of commutative local rings. This class of rings includes the important class of matrix rings over commutative local rings. In part 1 below we study some elementary properties of integral extensions and here we assume merely that the underlying ring is semi-local. In part 2 we discuss some questions of ideal theory for arbitrary local rings as well as for integral extensions. In a later paper we propose to utilize our results to study the deeper properties of these rings including a dimension theory for such rings. We are particularly indebted to the work of Nagata $[6,7,8,9]$ in the preparation of this paper.

\section{Part 1. Elementary properties}

If $R$ is a ring, $J(R)$ will designate the Jacobson radical of $R$. Throughout "ideal" will mean two-sided ideal.

(1.1) Def: If $R$ is a ring with identity, $R$ is said to be semi-local if:

(a) $R$ satisfies the maximum condition for left ideals;

(b) $R / J(R)$ satisfies the minimum condition for left ideals;

(c) $\bigcap_{n=0}^{\infty} J^{n}(R)=0, J^{o}(R)=R$.

If, in addition, $R$ satisfies:

(d) $J(R)$ is a unique maximal ideal in $R$, then $R$ is said to be a local ring. See [1].

Received June 3, 1958.

Revised July 15, 1958.

* See Bibliography at end of paper. 
(1.2) Def: Let $R$ be a ring with identity and $S$ a subring of the center of $R, 1 \in S . \quad R$ is said to be an integral extension of $S$ if $R$ satisfies the maximum condition for S-submodules. Clearly, every integral extension is a finite extension and if $S$ is Noetherian the two concepts coincide. If $R$ is an integral extension of $S$ and $r \in R$, then there exists a relation of the form $r^{n}+s_{n-1}$ $r^{n-1}+\ldots+s_{0}=0, s_{i} \in S$ all $i$. We shall assume the following properties of integral extensions.

(a) Every primitive ideal in $R$ contracts to a maximal ideal in $S$.

(b) If $P$ is prime in $S$ there is a prime ideal $P^{\prime}$ in $R$ such that $P^{\prime} \cap S=P$. If $P$ is maximal, $P^{\prime}$ is primitive.

(c) If $P^{\prime}$ is prime in $R, P^{\prime} \cap S$ is prime in $S$.

(d) $J(S) \cong J(R) \cap S$.

(e) If $R$ is an integral extension of a semi-local ring $S$, then $R$ is semilocal, and if $S$ is complete, $R$ is complete.

Proofs (a)-(c) can be found in [4] and proofs of (d) and (e) can be found in $[5]$.

We first show that if $R$ is an integral extension of a local ring $S$ then $S$ is a subspace of $R$ with respect to the topology of $R$ induced by powers of $J(R)$. The proof of this is contained in the following definition and lemmas:

(1.3) Def: If $R$ and $S$ are semi-local rings $S \subseteq R, R$ is said to be a concordant extension of $S$ if a sequence of elements $\left\{s_{i}\right\}, s_{i} \in S$ is regular in $S$ if and only if $\left\{s_{i}\right\}$ is regular in $R$.

(1.4) Lemma. If $R$ and $S$ are semi-local rings $S \subseteq R, R$ is a concordant extension of $S$ if and only if for any 2 positive integers $m, n$ we can find integers $p$ and $g \cong 0$ such that $J^{p}(S) \subseteq J^{m}(R) \cap S$ and $J^{n}(R) \cap S \subseteq J^{q}(S)$.

Proof. Follows directly from definition (1.3).

(1.5) Lemma. If $R$ is an integral extension of $S, R$ is a concordant extension of $S$.

Proof. Remark $(d)$ above shows $J(R) \cap S \supseteqq J(S)$ and, hence, $J^{n}(S) \cong J^{n}(R) \cap S$. Moreover, $\quad J^{m}(R) \cap S \supseteqq J^{m+1}(R) \cap S$ and $\bigcap_{n=0}^{\infty}\left[J^{n}(R) \cap S\right]=(0)$. It follows from [2, p. 695], that there exists a $p \geq 0$ such that $J^{p}(R) \cap S \subseteq J^{m}(S)$ for a given $m \geq 0$. Our result then follows from (1.4). 
(1.6) TheоRem. Let $S$ be a complete semi-local ring contained in the center of $R$ such that $\bigcap_{n=0}^{\infty}\left[J^{n}(S) \cdot R\right]=0$, then $[J(S) \cdot R] \cap S=J(S)$. If $R /[J(S) \cdot R]$ is an integral extension of $S / J(S)$, then $R$ is an integral extension of $S$ and a com. plete semi-local ring.

Proof. Since $1 \in R,[J(S) \cdot R] \cap S \supseteqq J(S)$. Since $S$ is commutative $J(S)=$ $\bigcap_{i=1}^{n} P_{i}$, where $P_{i}, i=1,2, \ldots, n$ are the maximal primes in $S$. If $[J(S) \cdot R] \cap$ $S \neq J(S)$, there is an $s \in S, s \in J(S) \cdot R, s \notin J(S)$. Then $s \notin P_{i}$, some $i$, say $P_{1}$. We have $P_{1}+s$ generates $S$ and $P_{1} R \cap S \supseteqq P_{1}+s$. Thus, $P_{1} R \cap S=S$ and, hence, $P_{1} R=R$ and for each integer $m, J^{m}(S) \cdot R=\left[\bigcap_{i=2}^{n} P_{i}\right]^{m} \cdot R$ which contradicts our assumption that $\bigcap_{n=0}^{\infty}\left[J^{n}(S) \cdot R\right]=0$. Thus, $[J(S) \cdot R] \cap S=J(S)$.

If $R /[J(S) \cdot R]$ is an integral extension of $S / J(S)$, then $R /[J(S) \cdot R]=$ $[S / J(S)]\left(\bar{u}_{1}, \ldots, \bar{u}_{n}\right)$ with $\bar{u}_{i} \in R /[J(S) \cdot R], i=1,2, \ldots n$. If $u_{i}, i=1,2$, $\ldots, n$ is a set of elements in $R$ such that $u_{i}$ maps on $\bar{u}_{i} \bmod [J(S) \cdot R]$ then $R=S\left(u_{1}, \ldots, u_{n}\right)$. In order to show this let $r \in R$. Form $n$ sequences $r_{i, j}$, $i=1,2, \ldots, n, j=0,1,2, \ldots$ as follows : $r_{i, 0}=0$, all $i$ and if $r_{i, k}$ has been defined for all $k \leqq m$ and each $i$ so that $r-\sum_{i=1}^{n} r_{i}, m u_{i} \in\left[J^{m}(S) \cdot R\right]$, then we have, $r-\sum_{i=1}^{n}\left(r_{i}, m \cdot u_{i}\right)=\sum_{t=1}^{h} \alpha_{t} z_{t}, \alpha_{t} \in R, z_{t} \in J^{m}(S)$ and further, $\alpha_{t}=\sum_{i=1}^{n}\left(a_{t, i} \cdot u_{t}\right)$ $\bmod [J(S) \cdot R], a_{t, i} \in S$. Thus, if we let $r_{i, m+1}=r_{i}, m+\sum_{t=1}^{h} a_{t, i} \cdot z_{t}$, the sequence $\left\{r_{i}, j\right\}$ is regular in $S$ for each $i$ and hence, has a limit $r_{i}$. Setting $r^{\prime}=\sum_{i=1}^{n}$ $\boldsymbol{r}_{i} \cdot u_{i}$, we have $\left(r^{\prime}-r\right) \in\left[J^{n}(S) \cdot R\right]$ for each $n$ and, hence, $r^{\prime}=r$ and $R=$ $S\left(u_{1}, \ldots, u_{n}\right)$. The completion of $R$ follows from the remarks following (1.2).

The following theorem proves that the class of integral extensions of semilocal rings have completions which are also semi-local.

(1.7) THEOREM. If $R$ is an integral extension of a semi-local ring $S$, then the completion $\bar{R}$ of $R$ is semi-local.

Proof. $\quad R$ is semi-local and has a completion $\bar{R}$. Now $S$ is a subspace as well as a subring of $R$ by (1.5) and, consequently, $S$ is a subspace of $\bar{R}$. Thus, the completion of $S, \bar{S}$, is its closure in $\bar{R}$. Thus, $\bar{R}=\bar{S}\left(u_{1}, \ldots, u_{n}\right)$ where $R=S\left(u_{1}, \ldots, u_{n}\right)$, for $S\left(u_{1}, \ldots, u_{n}\right)$ is a complete semi-local ring containing $R$ as a subring and subspace and it follows that $\bar{S}\left(u_{1}, \ldots, u_{n}\right)$ is contained in any complete ring containing $R$ as subring where the completion is taken 
relative to the powers of $J(R)$. The theorem then follows from the definition of this completion of $R$.

We conclude this section with the following lemma which further shows the analogy between integral extensions and commutative semi-local rings.

(1.8) Lemma. Let $R$ be an integral extension of a semi-local ring $S$ such that no element of $S$ is a zero-divisor in $\bar{R}$. If $u_{1}, \ldots, u_{n}$ are linearly independent over $S$ they are also linearly independent over $\bar{S}$. Moreover, if $t \in \bar{S}$ is a zero divisor in $\bar{R}$, it is also a zero divisor in $\bar{S}$.

Proof. Since $R$ satisfies the maximum condition on $S$-modules, it follows that $u_{1}, \ldots, u_{n}$ can be extended to a maximal set of linearly independent (over $S)$ elements which we can assume to be $u_{1}, \ldots, u_{n}$. There then exists an $s \in S$ such that $s R \cong S u_{1}+\ldots+S u_{n}, s \neq 0$. Suppose now that we have $\sum_{i=1}^{n}$ $s_{i} \cdot u_{i}=0, s_{i} \in \bar{S}$. For each $i, s_{i}=\lim _{j} s_{i j}, s_{i j} \in S$ and $\sum_{i=1}^{n}\left(s \cdot s_{i j} \cdot u_{i}\right) \in \sum_{i=1}^{n}\left(J^{j}(S) \cdot\right.$ $u_{i}$ ) for each $j=0,1,2, \ldots$ Since $\left\{u_{i}\right\}, i=1, \ldots, n$ are linearly independent over $S, s \cdot s_{i j}=a_{i j}, a_{i j} \in J^{j}(S)$ for each $i$ and $j$. Thus, $\left\{s \cdot s_{i j}\right\}, j=0,1,2, \ldots$ is a null sequence for each $i=1,2, \ldots, n$. Since $s$ is not a zero divisor in $\bar{R}$, $s_{i}=0, i=1,2, \ldots, n$.

Now, let $t$ be any element of $\bar{S}$ which is not a zero divisor in $\bar{S}$ and suppose $t r=0, r \in \bar{R}$. If $s$ is such that $s R \subseteq S u_{1}+\ldots+S u_{n}, s \in S, s \neq 0, s r=$ $\sum_{i=1}^{n}\left(s_{i} \cdot u_{i}\right), s_{i} \in \overline{\mathrm{S}}$. Hence, $\sum_{i=1}^{n}\left(t \cdot s_{i} \cdot u_{i}\right)=0$, and $t \cdot s_{i}=0, i=1,2, \ldots, n$. We then have $s_{i}=0, i=1,2, \ldots, n$ and, hence, $s \cdot r=0$ and $r=0$.

\section{Part II. Ideal theory and chain conditions}

Integral extensions of local rings satisfy a strong chain condition with respect to a certain class of ideals. We begin with a definition of this class of ideals.

(2.1) Def: An ideal $P$ in an arbitrary ring $R$ is said to be prime if $A \cdot B \subseteq P$ implies either $A \subseteq P$ or $B \subseteq P, A, B$, ideals of $R$. An ideal $Q$ in $R$ is said to be left primary if $A B \cong Q, B \notin Q$ implies $A^{n} \leqq Q$ for some integer $n>0$, $A, B$ ideals of $R$. If $Q$ is left primary, let $P$ be the join of all ideals $I$ of $R$ having the property that $I^{n} \leqq Q$ for some integer $n>0 . \quad P$ is an ideal of $R$ and if $R$ satisfies the maximum condition for left ideals, $P$ is a prime ideal, called the associated prime of $Q$. It follows that for some integer $m>0, P^{m} \subseteq Q$. 
The following shows that there is an abundance of left primary ideals in a local ring.

(2.2) Lemma. If $R$ is a local ring, $J(R)$ is a prime ideal and $J^{n}(R)$ is a left primary ideal with associated prime $J(R)$ for each integer $n>0$.

Proof. Since $J(R)$ is primitive, it is prime. For every proper ideal $I \subset R$, $I \subseteq J(R)$ and $I^{n} \subseteq J^{n}(R)$ for all $n>0$. Thus, $J^{n}(R)$ is left primary with associated prime for each integer $n>0$.

Cor. If $R$ is a local ring and $Q$ a left primary ideal with associated prime $J(R)$, then $J(R)$ is a unique prime for $Q$.

(2.3) Lemma. If $R$ is a local ring and $I$ an ideal of $R$, then $I$ is a left primary ideal with associated prime $J(R)$ if and only if $J^{n}(R) \subseteq I$ for some integer $n>0$.

Proof. If $I$ is left primary with associated prime $J(R)$, then $J^{n}(R) \subseteq I$, some integer $n>0$. Conversely if $J^{n}(R) \subseteq I \subseteq J(R)$, then $I$ is clearly left primary with associated prime $J(R)$.

Cor. If $R$ is a local ring and I a left primary ideal with associated prime $J(R)$, then $I$ is closed in $R$.

Proof. $J^{n}(R) \subseteq I$ by the lemma. Hence, $I+J^{m}(R)=I$ for all $m \geqq n$. Thus, $\bigcap_{i=0}^{\infty}\left[J^{i}(R)+I\right]=[J(R)+I] \cap\left[J^{2}(R)+I\right] \cap \ldots \cap\left[J^{n-1}(R)+I\right] \cap I=1$.

Thus, $I$ is closed.

In [1], we have shown that a necessary and sufficient condition that $R$ be a complete local ring is that $R=(C)_{n}$ - the ring of $n \times n$ matrices over a completely primary ring $C(C / J(C)$ is a division ring) where $C$ itself is a complete local ring. It is clear that $P$ is prime in $C$ if and only if $(P)_{n}$ is prime in $(C)_{n} ; Q$ is left primary in $C$ if and only if $(Q)_{n}$ is left primary in $(C)_{n} ; I_{1} \subseteq$ $I_{2} \subseteq \ldots$ in $C$ if and only if $\left(I_{1}\right)_{n} \subseteq\left(I_{2}\right)_{n} \subseteq \ldots$ in $(C)_{n}$, and similarly for descending chains. Thus, we reduce certain questions concerning complete local rings to questions concerning completely primary rings. The following lemma is the key in our reduction and is of some independent interest.

(2.4) Lemma. Let $R$ be a completely primary ring satisfying the maximum condition for left ideals. Then, for any integer $n \geqslant 1, J^{n}(R) / J^{n+1}(R)$ is a finite dimensional left vector space over $R / J(R)$. Moreover, if $J^{n}(R) \supseteqq T \supseteqq J^{n+1}(R)$ 
for a left ideal $T$ of $R$, then $T / J^{n+1}(R)$ is a finite dimensional subspace of $J^{n}(R) / J^{n+1}(R)$.

Proof. If $[r+J(R)] \in R / J(R),\left[t+J^{n+1}(R)\right] \in J^{n}(R) / J^{n+1}(R)$ define $[r+$ $J(R)] \cdot\left[t+J^{n+1}(R)\right]=\left[r t+J^{n+1}(R)\right] \in J^{n}(R) / J^{n+1}(R)$. Clearly this is well defined and $J^{n}(R) / J^{n+1}(R)$ is a left vector space over $R / J(R)$. Since $J^{n}(R)=$ $R\left(u_{1}, \ldots, u_{s}\right), u_{i} \in J^{n}(R), i=1,2, \ldots s$, every element of $J^{n}(R) / J^{n+1}(R)$ can be written in the form: $\bar{r}_{1} \bar{u}_{1}+\ldots+\bar{r}_{s} \bar{u}_{s}, \bar{r}_{i} \in R / J(R), \bar{u}_{i} \in J^{n}(R) / J^{n+1}(R)$. Thus, $J^{n}(R) / J^{n+1}(R)$ has finite left-dimension over $R / J(R)$. The remainder of the lemma follows immediately.

With the aid of the above lemma, we prove the following fundamental theorem.

$(2.5)^{*}$ THEOREm. Let $R$ be a completely primary ring satisfying the maximum condition for left ideals and let $Q$ be any left primary ideal in $R$ with associated prime $J(R)$. Then $R / Q$ satisfies the minimum condition for left ideals.

Proof. $J(R / Q)=J(R) / Q$ and $R / Q / J(R) / Q \cong R / J(R)$. We then have $R^{\prime}=$ $R / Q$ is completely primary and satisfies the maximum condition for left ideals. If we let $J^{\prime}=J\left(R^{\prime}\right)$ then $J^{\prime}$ is nilpotent of index of nilpotency $m$. It follows then from (2.4) that we have a composition series for $J^{\prime n} / J^{\prime n+1}$ as a left $R^{\prime}$. module for each $n>0$. Since $J^{\prime}$ is nilpotent of index $m$ combining composition series for $J^{\prime} / J^{\prime 2}, J^{\prime 2} / J^{\prime 3}, \ldots, J^{m-1} / J^{\prime m}$ we obtain a composition series for the left $R^{\prime}$ module $R^{\prime}$. It then follows from the Jordan Hölder theorem that $R / Q$ satisfies the minimum condition for left ideals.

(2.6) TheoRem. Let $R$ be a complete local ring and let $Q$ be a left primary ideal with associated prime $J(R)$, then $R / Q$ satisfies the minimum conditions for left ideals.

Proof. $R \cong(C)_{n}$ with $C$ completely primary. Moreover, there is a left primary ideal $I$ in $C$ such that $Q=(I)_{n}$ and the associated prime of $I$ is $J(C)$. Thus $R / Q \cong(C / I)_{n}$ and the result follows from(2.5) and our preliminary remarks. We remark that we can state a somewhat stronger 'result than (2.6) namely, if $R$ is isomorphic to $(C)_{n}$ and $C$ is completely primary with maximum

* The author wishes to thank the referee for suggestions which led to essential simplifications of $(2.5),(2.6)$ and $(2.7)$. 
conditions on left ideals, then if $Q$ is left primary with associated prime $J(R)$, $R / Q$ satisfies the minimum conditions on left ideals. (2.6) is the form in which the resuit is most useful for local rings.

We now state a form of (2.6) for integral extensions. Here we need not demand that the rings under consideration be complete.

(2.7) Theorem. Let $R$ be an integral extension of a local ring $S$. If $Q$ is any left primary ideal in $R$ which contracts to a $J(S)$-primary ideal in $S$, then $R / Q$ satisfies the minimum conditions on one-sided ideals.

Proof. Since $S$ in Noetherian and $Q \cap S$ is $J(S)$-primary $S /[Q \cap S]$ satisfies the minimum condition. $R / Q$ is an integral extension of $S /[Q \cap S]$ and is, moreover, a finitely generated right or left $S /[Q \cap S]$-module. It then follows that $R / Q$ satisfies the minimum condition on right or left $S_{/}[S \cap Q]$ submodules. Hence, $R / Q$ satisfies the minimum condition one one-sided ideals.

In view of the remarks following (1.2) the following is an immediate consequence of the theorem.

CoR. If $R$ is an integral extension of a local ring $S$ and $Q$ a left primary ideal in $R$ whose associated prime is primitive, then $R_{l}^{\prime} Q$ satisfies the minimum condition for two-sided ideals.

\section{BIBLIOGRAPHY}

[1] Batho, E. H., Non-commutative semi-local and local rings, Duke Math. Journal, vol. 24 (1957), pp. 163-172.

[2] Chevalley, C., On the theory of local rings, Annals of Mathematics, vol. 44 (1943), pp. 690-708.

[3] Cohen, I. S., On the structure and ideal theory of complete local rings, Transactions of the American Mathematical Society, vol. 59 (1946), pp. 54-106.

[4] Curtis, C. W., Non-commutative extensions of Hilbert Rings, Proceedings of the American Mathematical Society, vol. 4 (1953), pp. 945-955.

[5] Curtis, C. W., The Structure of non-semisimple algebras, Duke Math. Journal, vol. 21 (1954), pp. 79-86.

[6] Nagata, M., On the structure of complete local rings, Nagoya Mathematical Journal, vol. 1 (1950), pp. (63-70).

[7] Nagata, M., Seme remarks on local rings, Nagoya Mathematical Journal, vol. 6 (195.3), pp. $53-58$.

[8] Nagata, M., Some studies on semi-local rings, Nagoya Mathematical Journal, vol. 3 (1951), pp. 23-30.

[9] Nagata, M., On the theory of semi-local rings, Proceedings of the Japan Academy, vol. 26 (1950), pp. 131-140.

University of Rochester 